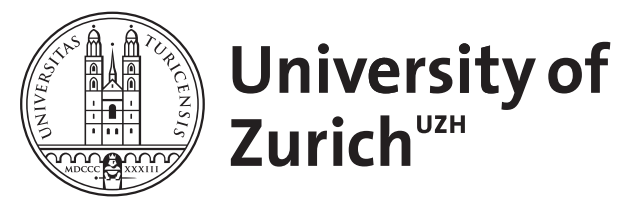

\title{
Diagnose von Weichteiltumoren in der Zytologie
}

\author{
Bode-Lesniewska, B
}

\begin{abstract}
Weichteilschwellungen sind das Symptom einer Vielzahl von neoplastischen und nichtneoplastischen Veränderungen. Sarkome gehören zu den selteneren Ursachen. Die Feinnadelpunktion hat sich in der Abklärung von Tumorrezidiven und Karzinommetastasen als minimal-invasive, kostengünstige und zuverlässige Methode bewährt. Auch in der Abklärung von Weichteiltumoren kann sie zu einer präzisen Diagnose führen. Voraussetzung ist eine enge multidisziplinäre Zusammenarbeit unter Einbeziehung klinischer, radiologischer und morphologischer Befunde. Für die zytologische Befundung sind Alter und Geschlecht sowie Topographie, Größe und Wachstumsgeschwindigkeit des Tumors wichtige Parameter. Reifungsgrad und Form der Zellen sowie Vorhandensein und Ausdifferenzierung der bindegewebigen Matrix bieten erste differenzialdiagnostische Hinweise und sind der Ausgangspunkt für immunzytochemische und molekularbiologische (FISH, RT-PCR) Zusatzuntersuchen. Diese Untersuchungen werden an Direktausstrichen, an mittels Zellblockmethode eingebettetem und an tiefgefrorenem Punktionsmaterial ausgeführt
\end{abstract}

DOI: https://doi.org/10.1007/s00292-007-0928-4

Other titles: Cytologic diagnosis of soft tissue tumors

Posted at the Zurich Open Repository and Archive, University of Zurich

ZORA URL: https://doi.org/10.5167/uzh-156159

Journal Article

Published Version

Originally published at:

Bode-Lesniewska, B (2007). Diagnose von Weichteiltumoren in der Zytologie. Der Pathologe, 28(5):368376.

DOI: https://doi.org/10.1007/s00292-007-0928-4 
Pathologe 2007 · 28:368-376

DOI 10.1007/s00292-007-0928-4

Online publiziert: 29. Juli 2007

(c) Springer Medizin Verlag 2007
B. Bode-Lesniewska

Abteilung Zytopathologie, Institut für Klinische Pathologie,

Universitätsspital Zürich, Schweiz

\section{Diagnose}

von Weichteiltumoren in der Zytologie

\section{Feinnadelpunktion von Weichteiltumoren}

Der hohe Stellenwert der Feinnadelpunktion (FNP) in der Abklärung von raumfordernden Prozessen unterschiedlicher Organe, insbesondere im Hinblick auf die Diagnostik von epithelialen Neoplasien, ist allgemein anerkannt. Die primären Weichteiltumoren gelten hingegen als wenig zugänglich für die zytologische Diagnostik, was sich in den letzten Jahren jedoch zunehmend geändert hat. Die Schwierigkeiten, die ein Zytopathologe auf diesem Gebiet antrifft, sind mannigfach: Einerseits ist es die häufig suboptimale Qualität des zur Verfügung stehenden Materials und das Fehlen klinischer Angaben, anderseits aber auch die geringe Erfahrung des Zytologen mit dieser äußerst heterogenen Gruppe von eher seltenen Tumoren. In der letzten WHOKlassifikation [10] werden über 100 unterschiedliche Weichteiltumorentitäten mit zahlreichen morphologischen Varianten aufgeführt. Weichteilsarkome stellen weniger als $1 \%$ aller malignen $\mathrm{Tu}$ moren dar. Die benignen Tumoren des Weichgewebes unter Berücksichtigung der Hämangiome und Lipome sind deutlich häufiger.

Indikationen zur zytologischen Abklärung sind neu aufgetretene und an Größe zunehmende Weichteilknoten bei Patienten mit und ohne Tumorerkrankungen in der Vorgeschichte. Ziel der Untersuchungen ist die Unterscheidung zwischen entzündlichen Prozessen, benignen Tumoren und malignen/malignitätssuspekten Geschwülsten.
Über beeindruckende Ergebnisse der FNP bei Weichteilneoplasien wird vor allem aus Schweden (Lund; [5, 6, 7, 8, 9]) und Frankreich (Institut Curie, Paris; [17, 18, 19, 20, 21, 22]) berichtet. In diesen Zentren ist die FNP bei Verdacht auf einen Weichteiltumor ein integrales Element der multidisziplinären Abklärungsuntersuchungen. Das allgemeine Verständnis der Vorgehensweise bei der Beurteilung der zytologischen Proben aus Weichteilläsionen ist für jeden diagnostisch tätigen Zytopathologen wichtig. Die technischen Fortschritte der letzten Jahre machen die Übernahme der diagnostischen und prognostischen Zusatzuntersuchungen der Histologie (Immunhistochemie, Fluoreszenz-insitu-Hybridisierung/FISH, RT-PCR, Sequenzierung) und ihre Anwendung an zytologischen Proben selbstverständlich. Das Vorgehen folgt der derzeitigen allgemeinen Tendenz in Richtung minimal-invasiver Abklärungsuntersuchungen und erfüllt die gesundheitspolitische Forderung, die Kosten ohne Qualitätseinbuße zu minimieren. Die in der Regel mit kaum oder nur minimalen Komplikationen behaftete FNP stellt eine technisch wenig anspruchsvolle, ambulant durchführbare Alternative dar im Vergleich zu einer offenen Biopsie mit deutlich höheren Risiken (Anästhesie, Nachblutung, Infektion oder Kontamination des Stichkanals). Die geringeren Kosten und die schnelleren Verarbeitungszeiten gehören ebenfalls zu den unbestreitbaren Vorteilen der FNP [1, 2, 23, 24].

In den Händen erfahrener Zytologen erreichen Sensitivität und Spezifität der FNP im Hinblick auf die Unterscheidung benigner von malignen Läsionen nahe- zu 95\%. Die „falsch-positive“ Rate wird in der Literatur mit $0-5 \%$, die „falsch-negative" Rate mit 2-15\% angegeben [2, 12, 24]. Die falsch-negativen Diagnosen können meistens auf suboptimales Material oder Fehlinterpretationen zurückgeführt werden und sind in der Regel nicht mit schwerwiegenden Folgen behaftet, da eine klinisch suspekte Läsion ohnehin weiter morphologisch abgeklärt wird.

\section{Entnahmetechnik}

Entscheidend für den diagnostischen Erfolg einer FNP (selbstverständlich nicht nur auf dem Gebiet der Weichteilpathologie) ist die korrekte Entnahmetechnik. Ähnlich wie bei der Auswahl der Biopsiestelle an den Extremitäten, muss der transkutane FNP-Stichkanal unter Vermeidung einer eventuellen Kontamination der nicht vom Tumor betroffenen Kompartimente angelegt werden. In der Regel bedeutet dies, dass der kürzeste Weg zwischen dem Tumor und der Haut - am besten in der Absprache mit dem beteiligten Chirurgen/Orthopäden - gewählt wird. Die Punktion wird mit einer dünnen Nadel $(22-25 \mathrm{G})$ durchgeführt. Von Vorteil ist die Zuhilfenahme eines Halters (z. B. Cameco ${ }^{\circ}$ ). Das Material wird fächerförmig (um die Materialentnahme aus möglichst großem Tumorvolumen zu gewährleisten) mit schneidenden Bewegungen unter dem nach Bedarf angelegten Sog über etwa 20-30 s gewonnen. Die Materialasservierung in entsprechenden Medien für diagnostische Zusatzmethoden ist von entscheidender Bedeutung. Dieses Ziel lässt sich erfahrungsgemäß am bes- 


\begin{tabular}{|c|c|c|c|c|c|}
\hline Spindelzellen & Myxoide Matrix & Pleomorphe Zellen & Runde Zellen & Epitheloide Zellen & $\begin{array}{l}\text { Differenziertes } \\
\text { Gewebe }\end{array}$ \\
\hline $\begin{array}{l}\text { - Neurofibrom } \\
\text { - Schwannom } \\
\text { - Noduläre Fasziitis } \\
\text { - Proliferative Fasziitis } \\
\text { - Proliferative Myositis } \\
\text { - Tenosynovialer } \\
\text { Riesenzelltumor } \\
\text { - Leiomyom } \\
\text { - Fibromatose (Des- } \\
\text { moidtumor) }\end{array}$ & $\begin{array}{l}\text { - Ganglion, Bursa } \\
\text { - Myxoma } \\
\text { - intramuskuläres } \\
\text { - juxtaartikuläres } \\
\text { - Noduläre Fasziitis } \\
\text { - Neurofibrom } \\
\text { - Spindelzelllipom } \\
\text { - Lipoblastom }\end{array}$ & $\begin{array}{l}\text { - Pleomorphes Lipom } \\
\text {-Proliferative Myositis } \\
\text {-Proliferative Fasziitis }\end{array}$ & & $\begin{array}{l}\text { - Granularzelltumor } \\
\text { - Proliferative Myositis }\end{array}$ & $\begin{array}{l}\cdot \text { Lipom } \\
\cdot \text { Chondrom }\end{array}$ \\
\hline $\begin{array}{l}\text {-Solitärer fibröser } \\
\text { Tumor }\end{array}$ & & · Paragangliom & & $\begin{array}{l}\text { - Paragangliom } \\
\text { · Epitheloides } \\
\text { · Hämangioendo- } \\
\text { theliom }\end{array}$ & \\
\hline $\begin{array}{l}\text { - Gastrointestinaler } \\
\text { Stromatumor } \\
\text { - DFSP } \\
\text { - MPNST } \\
\text { - Synovialsarkom } \\
\text { - Leiomyosarkom } \\
\text { - Dedifferenziertes } \\
\text { Liposarkom } \\
\text { - Myofibroblastisches } \\
\text { Sarkom } \\
\text { - Embryonales } \\
\text { Rhabdomyosarkom } \\
\text { - Kaposi-Sarkom }\end{array}$ & $\begin{array}{l}\cdot \text { Myxoides Liposarkom } \\
\cdot \text { Myxofibrosarkom } \\
\cdot \text { Myxoides Chondro- } \\
\text { sarkom } \\
\text { - Niedrig malignes } \\
\text { fibromyxoides Sarkom }\end{array}$ & $\begin{array}{l}\text { - Undifferenziertes } \\
\text { pleomorphes Sarkom } \\
\text { - Pleomorphes } \\
\text { Leiomyosarkom } \\
\text { - Pleomorphes Rhab- } \\
\text { domyosarkom } \\
\text { - Pleomorphes Lipo- } \\
\text { sarkom } \\
\text { - Pleomorphes MPNST } \\
\text { - Angiosarkom } \\
\text { - Extraskeletales } \\
\text { Osteosarkom }\end{array}$ & $\begin{array}{l}\text { - Alveoläres } \\
\text { Rhabdomyosarkom } \\
\text { - Neuroblastom } \\
\text { - Ewing-Sarkom/PNET } \\
\text { - Synovialsarkom } \\
\text { - Rundzelliges } \\
\text { Liposarkom } \\
\text { - Mesenchymales } \\
\text { Chondrosarkom } \\
\text { - DSRCT } \\
\text { - Rhabdoidtumor } \\
\text { - Pigmentierter, neuro- } \\
\text { ektodermaler Tumor } \\
\text { des Kinderalters }\end{array}$ & $\begin{array}{l}\text { - Epitheloides Sarkom } \\
\text { - Epitheloides } \\
\text { Angiosarkom } \\
\text { - Epitheloides } \\
\text { Leiomyosarkom } \\
\text { · Epitheloides MPNST } \\
\text { - Alveoläres } \\
\text { Weichteilsarkom } \\
\text { - Klarzellsarkom } \\
\text { - Rhabdoidtumor }\end{array}$ & $\begin{array}{l}\text { - Gut differenziertes } \\
\text { Liposarkom } \\
\text { - Niedrig malignes } \\
\text { Chondrosarkom }\end{array}$ \\
\hline \multicolumn{6}{|c|}{$\begin{array}{l}\text { Der obere Teil der Tabelle umfasst gutartige, nichtmetastasierende Tumoren, der untere Teil hingegen eindeutig maligne Neoplasien. Im mittleren Teil werden } \\
\text { Läsionen mit morphologisch schwer einschätzbarem Metastasierungspotenzial aufgelistet. } \\
\text { DFSP Dermatofibrosarcoma protuberans; MPNST maligner peripherer Nervenscheidentumor; PNET primitiver neuroektodermaler Tumor; DSRCT desmoplas } \\
\text { tischer klein- und rundzelliger Tumor. } \\
\text { Grundregel:Zuerst eine Manifestation eines Karzinoms, Melanoms, Lymphoms oder Mesothelioms ausschließen! }\end{array}$} \\
\hline
\end{tabular}

ten erreichen, wenn der Eingriff entweder vom Zytopathologen selbst, häufig unter Ultraschallsteuerung, durchgeführt wird (die von uns bevorzugte Variante; vgl. - Abb. 2, 5) oder wenn die Punktion in Anwesenheit eines Zytologen oder eines speziell ausgebildeten Zytotechnikers erfolgt. Bei intrathorakal oder intraabdominell gelegenen Tumoren muss eine CT-gesteuerte transkutane oder transendoskopische ultraschallgesteuerte FNP durchgeführt werden (vgl. • Abb. 1, 3, 4). Häufig, besonders bei kollagenarmen, zellreichen Läsionen, gelingt die Entnahme von ausreichenden Gewebemengen bereits in Rahmen einer einzigen Punktion. Bei Bedarf lassen sich in der gleichen Sitzung weitere Punktionen durchführen die FNP ist für den Patienten wenig belastend. Eine Lokalanästhesie ist bei oberflächlichen, d. h. ohne CT-Steuerung erreichbaren, Knoten nicht notwendig.

\section{Präparateherstellung und Materialasservierung}

Primär werden 3-5 Direktausstriche angefertigt, die z. T. alkoholfixiert und $\mathrm{z}$. T. luftgetrocknet werden. Die feucht fixierten Ausstriche werden nach Papanicolaou, die luftgetrockneten mit DiffQuick gefärbt. Die Papanicolaou-Färbung erlaubt eine bessere Beurteilung der Kerneigenschaften, während die Diff-Quick-Methode die Eigenschaften der extrazellulären Grundsubstanz hervorhebt. Mittels der Diff-Quick-Färbung (Zeitaufwand weniger als 1 Minute) lässt sich zusätzlich schnell beurteilen, ob ausreichend diagnostisches Material erfasst wurde.

Die Direktausstriche eignen sich sehr gut für FISH-Untersuchungen, da bei dreidimensional erhaltenen Zellen alle Kerne das vollständige genetische Material enthalten. Das Restmaterial des Punk- tats wird für die Herstellung eines Formalin-fixierten, Paraffin-eingebetteten Zellblocks (vgl. - Abb. 1, 2, 4) eingesetzt. Hierbei ist die Verwendung von hämolysierenden Flüssigkeiten (z. B. Cytolyt, Cytyc $^{\circledast}$ ) von Vorteil.

Die Anwendung von Dünnschichtmethoden in der Zytologie der Weichteilneoplasien wird nicht empfohlen, weil damit besonders bei primären Weichteiltumoren wichtige Details des Tumoraufbaus und über die extrazelluläre Grundsubstanz verloren gehen [3]. Ein Teil des Punktionsmaterials kann nach Bedarf schockgefroren oder für die Tumorzytogenetik gewonnen werden [14]. Bei Verdacht auf einen entzündlichen Prozess kann problemlos eine weitere Probe steril für mikrobiologische bzw. mykobakteriologische Untersuchungen entnommen werden. 
Pathologe 2007 · 28:368-376 DOI 10.1007/s00292-007-0928-4

(C) Springer Medizin Verlag 2007

\section{B. Bode-Lesniewska \\ Diagnose von Weichteiltumoren in der Zytologie}

\section{Zusammenfassung}

Weichteilschwellungen sind das Symptom einer Vielzahl von neoplastischen und nichtneoplastischen Veränderungen. Sarkome gehören zu den selteneren Ursachen. Die Feinnadelpunktion hat sich in der Abklärung von Tumorrezidiven und Karzinommetastasen als minimal-invasive, kostengünstige und zuverlässige Methode bewährt. Auch in der Abklärung von Weichteiltumoren kann sie zu einer präzisen Diagnose führen. Voraussetzung ist eine enge multidisziplinäre Zusammenarbeit unter Einbeziehung klinischer, radiologischer und morphologischer Befunde. Für die zytologische Befundung sind Alter und Geschlecht sowie Topographie, Größe und Wachstumsgeschwindigkeit des Tumors wichtige Parameter. Reifungsgrad und Form der Zellen sowie Vorhandensein und Ausdifferenzierung der bindegewebigen Matrix bieten erste differenzialdiagnostische Hinweise und sind der Ausgangspunkt für immunzytochemische und molekularbiologische (FISH, RT-PCR) Zusatzuntersuchen. Diese Untersuchungen werden an Direktausstrichen, an mittels Zellblockmethode eingebettetem und an tiefgefrorenem Punktionsmaterial ausgeführt.

\section{Schlüsselwörter}

Feinnadelpunktion · Weichteiltumor · Sarkom · Immunhistochemie · Fluoreszenz-insitu-Hybridisierung (FISH)

\section{Cytologic diagnosis of soft tissue tumors}

\section{Abstract}

Soft tissue swellings represent common clinical signs of a variety of neoplastic and nonneoplastic lesions. Sarcomas are rarely the cause. Fine needle biopsy as a minimally invasive, economic and accurate method is well suited for the diagnostics of recurrent and metastatic diseases. Cytologic diagnosis of primary soft tissue tumors is also feasible but requires close collaboration with other medical disciplines and incorporation of clinical, radiological and morphologic findings. Clinical data such as age, gender, size and topography are also important parame- ters. Differentiation of the cells and the properties of the extracellular matrix offer initial indications for the differential diagnosis and are the starting point for immunohistochemical and molecular analyses (FISH, RT-PCR). These analyses can be performed on cytological smears, on paraffin-embedded cell blocks on snap frozen material.

\section{Keywords}

Fine needle biopsy · Soft tissue tumor · Sarcoma $\cdot$ Immunohistochemistry $\cdot$ FISH

\section{Zytomorphologische Klassifikation des Punktats}

Als Faustregel gilt in der Diagnostik einer Weichteilschwellung, dass ein entzündlicher Prozess, eine Melanom-, Karzinom- oder Lymphommanifestation ausgeschlossen wird, ehe man einen primären Weichteiltumor in Erwägung zieht. Die Interpretation des Punktats eines primären Weichteiltumors wird durch eine strukturierte Vorgehensweise, die auf dem zytomorphologischen Grundmuster der Direktausstriche basiert, erleichtert [1, 13, 23]. Verschiedene Autoren schlugen die Einordnung der Punktate in unterschiedliche zytoarchitektonische Gruppen vor; sie lassen sich aufgrund eigener Erfahrungen in der Praxis am besten in $6 \mathrm{Ka}-$ tegorien zusammenfassen (• Tab. 1; [12, $13,16,23])$.

Die große Spannbreite in der morphologischen Differenzierung einzelner Entitäten der Weichteilneoplasien führt dazu, dass der gleiche Tumortyp in der Differenzialdiagnose mehr als einem Grundmuster zugeordnet werden kann, wie z. B. das Neurofibrom (myxoides oder spindelzelliges Grundmuster) oder das Synovialsarkom (spindelzelliges oder rundzelliges Grundmuster). Die an den Direktausstrichen erhobenen Befunde werden mit den Befunden an "Mikrobiopsien“ in Zellblockpräparaten verglichen (vgl. - Abb. 1, 2, 4). Beides, ergänzt durch die Ergebnisse der Zusatzuntersuchungen, fließt in die Endbeurteilung mit ein.

\section{Schlüsselkriterien}

\section{Spindelzellen}

Das häufigste morphologische Bild bei zytologischen Untersuchungen primärer Weichteilläsionen ist eine spindelige Zellpopulation unterschiedlicher Zelldichte und Atypiegrades. Diese zytoarchitektonische Gruppe ist die umfangreichste Kategorie mit einer ausgesprochenen Vielfalt der differenzialdiagnostisch infrage kommenden Läsionen. Die Differenzialdiagnose umfasst nicht nur sekundäre oder primäre, benigne und maligne WeichteiIneoplasien sondern auch reaktive bzw. pseudosarkomatöse Prozesse. Die klinische und bildgebende Korrelation wie 
Abb. 1 Kleines retroperitoneales Schwannom bei einer 54-jährigen Frau. a Transduodenale Endosonographie. b Spindelzellige blande Zellen eingebettet in faserreicher Grundsubstanz im Direktausstrich einer endoskopischer FNP (Papanicolaou-Färbung, Ver-

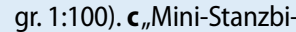
opsie-Zylinder" im Zellblock der FNP (HE-Färbung, Vergr. 1:100) mit typischem Erscheinungsbild und starker immunhistochemischer Expression des S100-Proteins (d; Vergr. 1:100)
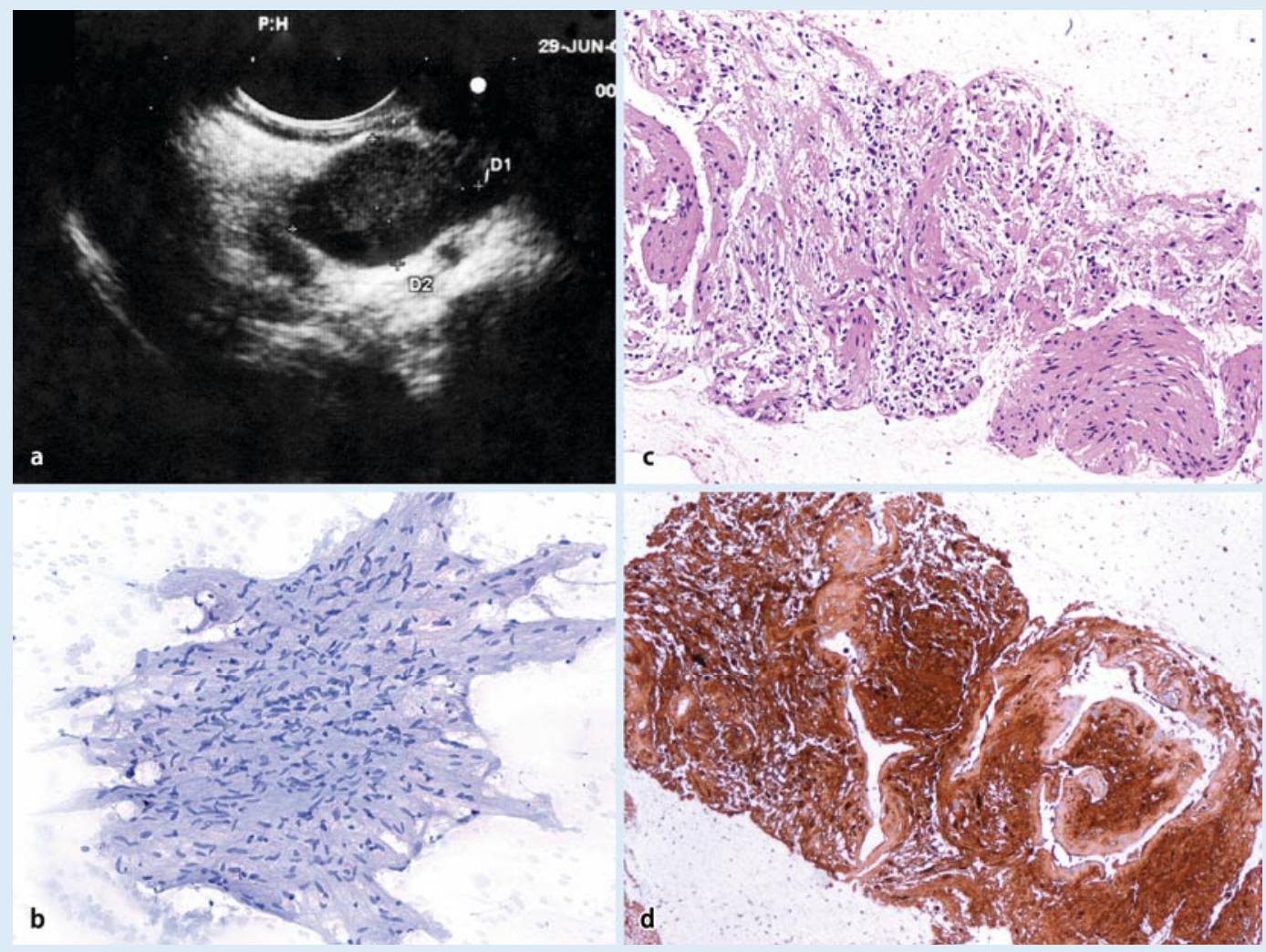

Abb. 2 Langsam über 2 Jahre wachsendes extraskeletales myxoides Chondrosarkom in der Knöchelgegend (a) einer 31-jährigen Patientin. b MRT: ausgedehnte Raumforderung mit Befall des Sehnenapparats und Ausdehnung zwischen Subkutis und Periost. c Direktausstrich mit reichlicher chondroider Grundsubstanz, die sich in der Diff-Quick-Färbung metachrom rot anfärbt (Vergr. 1:400). d Papanicolaou-Färbung (Vergr. 1:400): monomorphe Population mittelgroßer Zellen ohne Pleomorphie. e Zellblockmaterial (HE-Färbung, Vergr. 1:400): Nester und Trabekel blander Zellen, eingebettet in myxochondroider Grundsubstanz
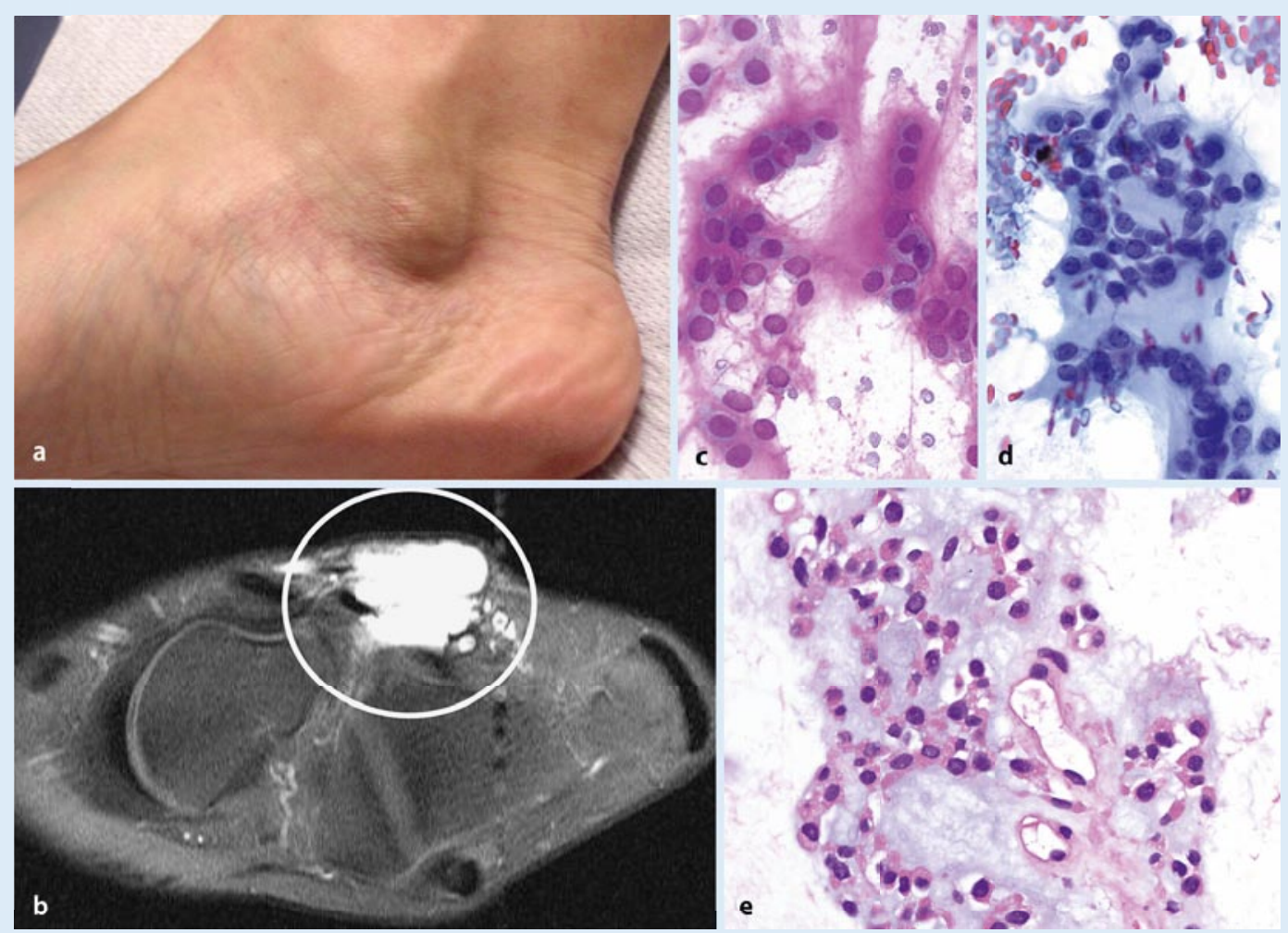

auch die Anwendung der diagnostischen Zusatzmethoden ist äußerst wichtig, da Läsionen aus dieser Gruppe mit einem hohem Risiko der Fehlbeurteilung behaftet sind [12].
Allgemein gilt, dass benigne Tumoren eher zellarme Punktate mit kompakten Gruppen von kleinen, monomorphen Zellen ergeben, während das Material maligner Neoplasien zahlreiche, dyskohäsive, pleomorphe und mitotisch aktive Zellen enthält. Der topographische Zusammenhang mit größeren Nerven lenkt die differenzialdiagnostischen Überlegungen zu den peripheren Nervenscheidentumo- 


\begin{tabular}{llll}
\hline $\begin{array}{l}\text { Tab. } 2 \text { Wichtige Weichteilsarkome mit diagnostisch relevanten zytogenetischen Verän- } \\
\text { derungen }\end{array}$ & Zytogenetische Aberration & Beteiligte Gene \\
\hline Tumor & $\mathrm{t}(11 ; 22)$ & EWS-FLI1 \\
Ewing-Sarkom/PNET & $\mathrm{t}(21 ; 22)$ & EWS-ERG \\
\hline Alveoläres Rhabdomyosarkom & $\mathrm{t}(2 ; 13)$ & PAX3-FKHR \\
& $\mathrm{t}(1 ; 13)$ & PAX7-FKHR \\
\hline Myxoides/rundzelliges Liposarkom & $\mathrm{t}(12 ; 16)$ & FUS-CHOP \\
& $\mathrm{t}(12 ; 22)$ & EWS-CHOP \\
\hline Myxoides extraskeletales Chondrosarkom & $\mathrm{t}(9 ; 22)$ & CHN-EWS \\
& $\mathrm{t}(9 ; 17)$ & CHN-RBP56 \\
& $\mathrm{t}(9 ; 15)$ & CHN-TCF12 \\
\hline Synovialsarkom & $\mathrm{t}(\mathrm{X} ; 18)$ & SYT-SSX1 \\
& & SYT-SSX2 \\
\hline Dermatofibrosarcoma protuberans & $\mathrm{t}(17 ; 22)$ & COLA1A1- \\
& & PDGFB \\
\hline Kongenitales Fibrosarkom & $\mathrm{t}(12 ; 15)$ & ETV5-NTRK3 \\
\hline Desmoplastischer klein- und rundzelliger Tumor & $\mathrm{t}(11 ; 22)$ & WT1-EWS \\
\hline Alveoläres Weichteilsarkom & $\mathrm{t}(\mathrm{X} ; 17)$ & ASPL-TFE3 \\
\hline Klarzellsarkom & $\mathrm{t}(12 ; 22)$ & ATF1-EWS \\
& $\mathrm{t}(2 ; 12)$ & CREB1-EWS \\
\hline Kursiv markiert: kommerziell erhältliche FISH-Sonden. & \\
\hline PNET: primitiver neuroektodermaler Tumor. & & \\
\hline
\end{tabular}

ren, während eine intraabdominelle Lokalisation die Diagnose eines gastrointestinalen Stromatumors (GIST) favorisieren lässt. Eine periartikuläre Lokalisation eines Tumors bei einem jungen Patienten macht ein Synovialsarkoms wahrscheinlich, während bei einer großen, tief liegenden Raumforderung am Oberschenkel eines älteren Patienten ein dedifferenziertes Liposarkom bzw. ein Leiomyosarkom infrage kommt.

Starke immunhistochemische Positivität für S1oo bei einem spindelzelligen Tumor (nach Ausschluss einer Melanommanifestation) ist bei den nichtepitheloiden malignen peripheren Nervenscheidentumoren (MPNST) ungewöhnlich und spricht für einen benignen neurogenen Tumor (• Abb. 1). Die immunzyto- bzw. immunhistochemisch nachgewiesene Expression der myogenen Marker (Aktin, Desmin, Myogenin, MyoD1) lässt eine leiomyomatöse bzw. rhabdomyomatöse Differenzierung vermuten. Die Expression von CD117 im entsprechenden klinischen Kontext belegt die $\mathrm{Di}$ agnose eines GIST, während der Nachweis einer SYT-SSX-Translokation für die Diagnose eines Synovialsarkoms beweisend ist (• Tab. 2; vgl. $\bullet$ Abb. 4).

\section{Myxoide Grundsubstanz}

Die Erkennung dieser zytomorphologischen Kategorie basiert nicht auf den Zellen, sondern auf der reichlich im Hintergrund vorhandenen myxoiden Grundsubstanz, die sich charakteristisch in der Papanicolaou-Färbung amorph grünlich bis blau darstellen lässt $[15,29]$. Die Differenzialdiagnose umfasst einige Entitäten, deren Unterscheidung aufgrund der in der Grundsubstanz eingebetteten Zellen unter Berücksichtigung der klinischen und bildgebenden Zusammenhänge erfolgt.

Eine praktisch azelluläre mukoide Grundsubstanz ist bei einem Ganglioninhalt zu erwarten, während spärliche, blande, kleine Spindelzellen ein intramuskuläres oder juxtaartikuläres Myxom vermuten lassen. Auffallend verzweigte, zarte Kapillaren auf myxoidem Hintergrund, begleitet von perivaskulär betonten, monomorphen, ovalären, kleinen Zellen in einer tief liegenden Läsion am Oberschenkel lassen die Diagnose eines myxoiden Liposarkoms annehmen. Diese Verdachtsdiagnose lässt sich durch den mittels FISH bzw. RT-PCR erfolgten Nachweis der diagnostischen Translokation (- Tab. 2) bestätigen und kann zu der adäquaten Therapie führen.
Im Gegensatz zum myxoidem Liposarkom zeigt das myxoide (extraskeletale) Chondrosarkom (- Abb.2) eine charakteristische, leuchtend rote, metachrome Anfärbbarkeit in einer Diff-Quick-Färbung am luftgetrockneten Ausstrich. Die in der Grundsubstanz eingelagerten Zellen sind ebenfalls eher monomorph und bland. Im Gegensatz dazu zeigt das Myxofibrosarkom eine auffallende Pleomorphie der auf einem myxoiden Hintergrund sichtbaren Zellen. In der Studie von Kilpatrick [15] konnte zytologisch in 13 von 16 (81\%) Patienten mit myxoiden Sarkomen ein genauer Sarkomtyp bestimmt werden.

\section{Pleomorphe Zellen}

Die Anwesenheit von zahlreichen, meist großen und unregelmäßig gestalteten Zellen lässt eine Weichteilschwellung in die Gruppe der pleomorphzelligen Läsionen einordnen. Dieses Grundmuster ist meist im Untersuchungsmaterial von älteren Patienten zu erwarten und wirft eine relativ breite Differenzialdiagnose von überwiegend hoch malignen Tumoren auf. Als erstes gilt es, eine Manifestation eines wenig differenzierten (großzelligen) Karzinoms, eines malignen Melanoms bzw. eines anaplastischen großzelligen Lymphoms auszuschließen. Dies ist in der Regel anhand der Klinik und durch den Einsatz von Antikörpern gegen Zytokeratine, melanozytäre (S10o, HMB45, Melan A) und lymphatische $\left(\mathrm{CD}_{45}, \mathrm{CD}_{3} \mathrm{O}, \mathrm{ALK}\right.$, $\mathrm{CD}_{2} \mathrm{O}, \mathrm{CD}_{3}$ ) Marker zu erreichen. Insbesondere bei Tumoren des Oberschenkels oder des Retroperitoneums muss an primäre Weichteilsarkome gedacht werden, und zwar in erster Linie an ein dedifferenziertes Liposarkom mit hochgradig maligner sarkomatöser Komponente (Positivität für MDM 2 und/oder CDK4;

\section{- Abb. 3).}

Parallel kommt auch ein myogenes Sarkom vom Typ eines hoch malignen Leiomyosarkoms (Positivität für Aktin und/ oder Desmin) oder ein pleomorphzelliges Rhabdomyosarkom (Positivität für Desmin, Myogenin, MyoD1) infrage. Weitere differenzialdiagnostische Optionen in dieser Gruppe stellen das pleomorphzellige Liposarkom (bizarre, pleomorphe Lipoblasten), das extraskeletale Osteosarkom 

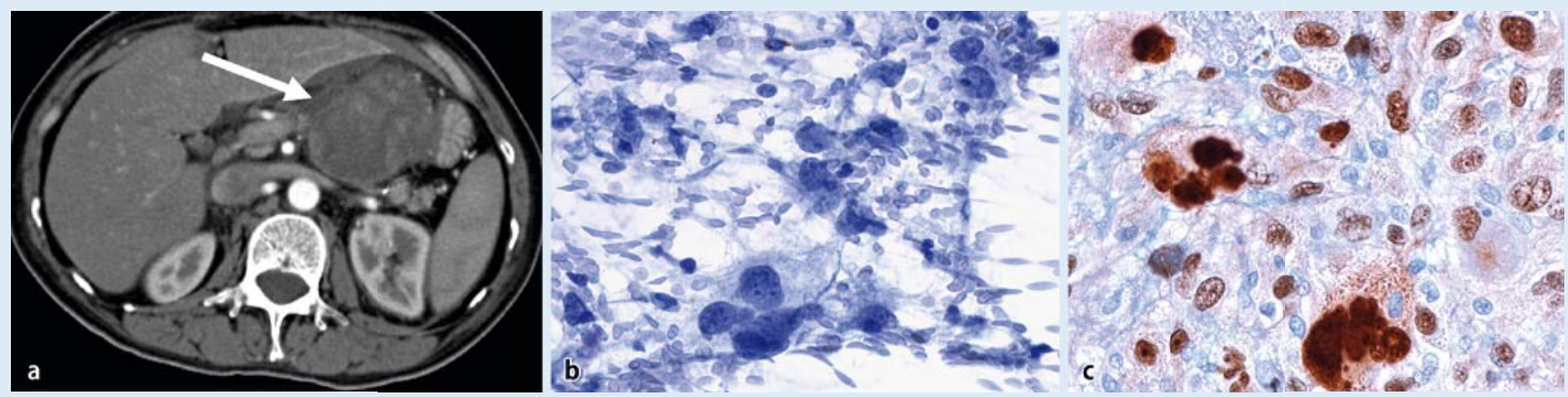

Abb. $3 \Delta$ Dedifferenziertes Liposarkom bei einer 51-jährigen Patientin. a CT des Abdomens: große intraabdominelle/retroperitoneale Raumforderung (Pfeil). b Direktausstrich (Vergr.1:400) der transduodenalen endosonographischen FNP: viele pleomorphe, große maligne Zellen, negativ reagierend in einer Immunzytochemie für Zytokeratin AE1/AE3. c Immunhistochemische MDM-2-Expression am Resektionspräparat mit deutlicher neukleärer Positivität (Vergr. 1:400)
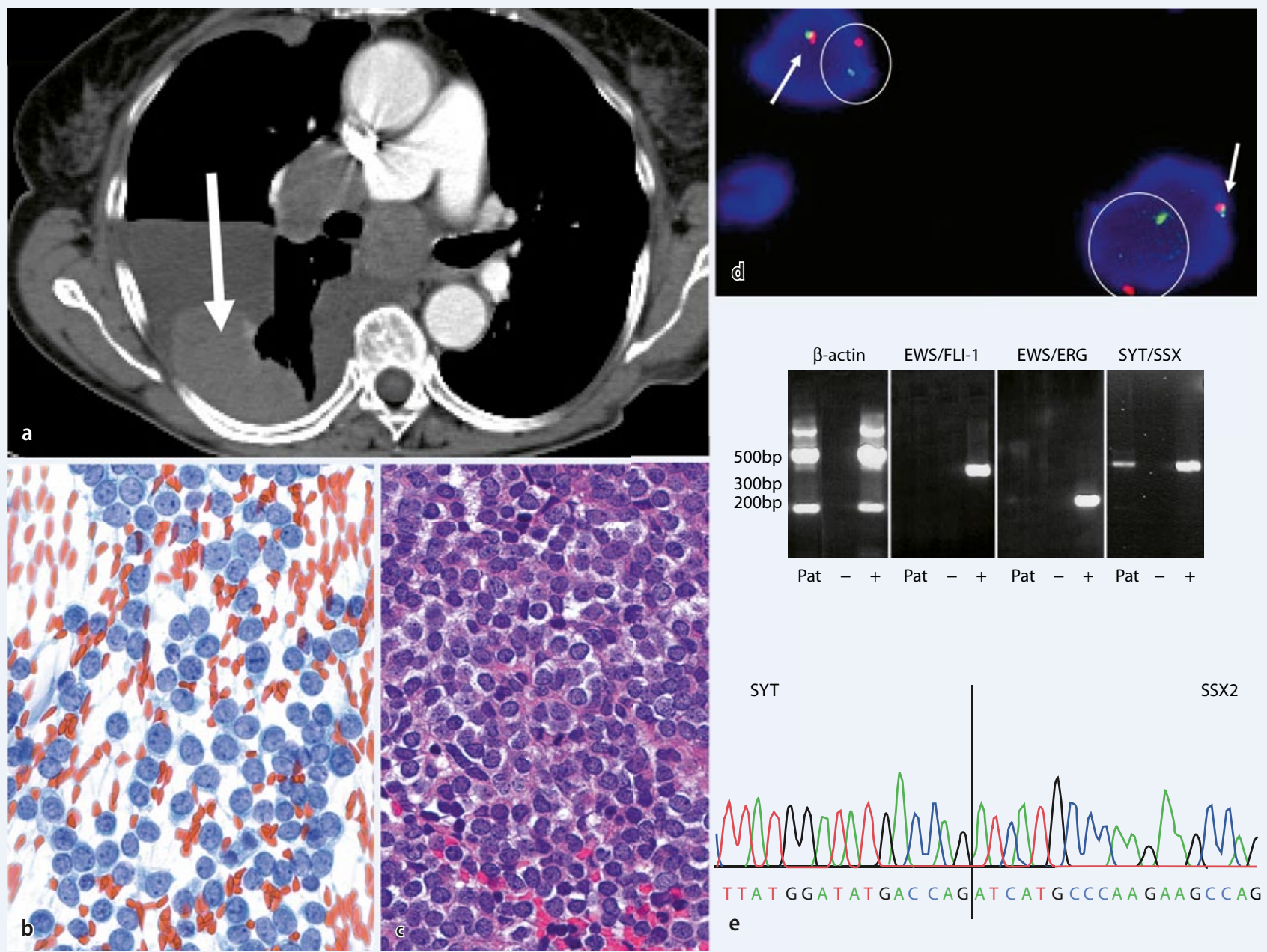

Abb. $4 \Delta$ a CT-gesteuerte transkutane FNP eines wenig differenzierten Synovialsarkoms der Pleura (Pfeil) einer 78-jährigen Patientin mit rezidivierenden Pleuraergüssen. b Direktausstrich (Papanicolaou-Färbung, Vergr. 1:400): monomorphe rundzellige Zellpopulation. c Im Zellblock (HE-Färbung, Vergr. 1:400): wenig differenzierte „klein-, blau- und rundzellige“ Neoplasie. d FISH-Untersuchung mit einer „Break-apart-SYT-Gen-Sonde“ (Vysis): Nachweis eines fusionierten (Pfeil) und eines getrennten (Kreis) Signals pro Kern, diagnostisch für ein Synovialsarkom. e RT-PCR-Reaktion: Fusionsprodukt SYT/SSX positiv und Translokationen EWS/FLI1, EWS/ERG negativ. Direkte Sequenzierung des Fusionsproduktes mit dem Typ SYT/SSX2 der Synovialsarkomtranslokation 


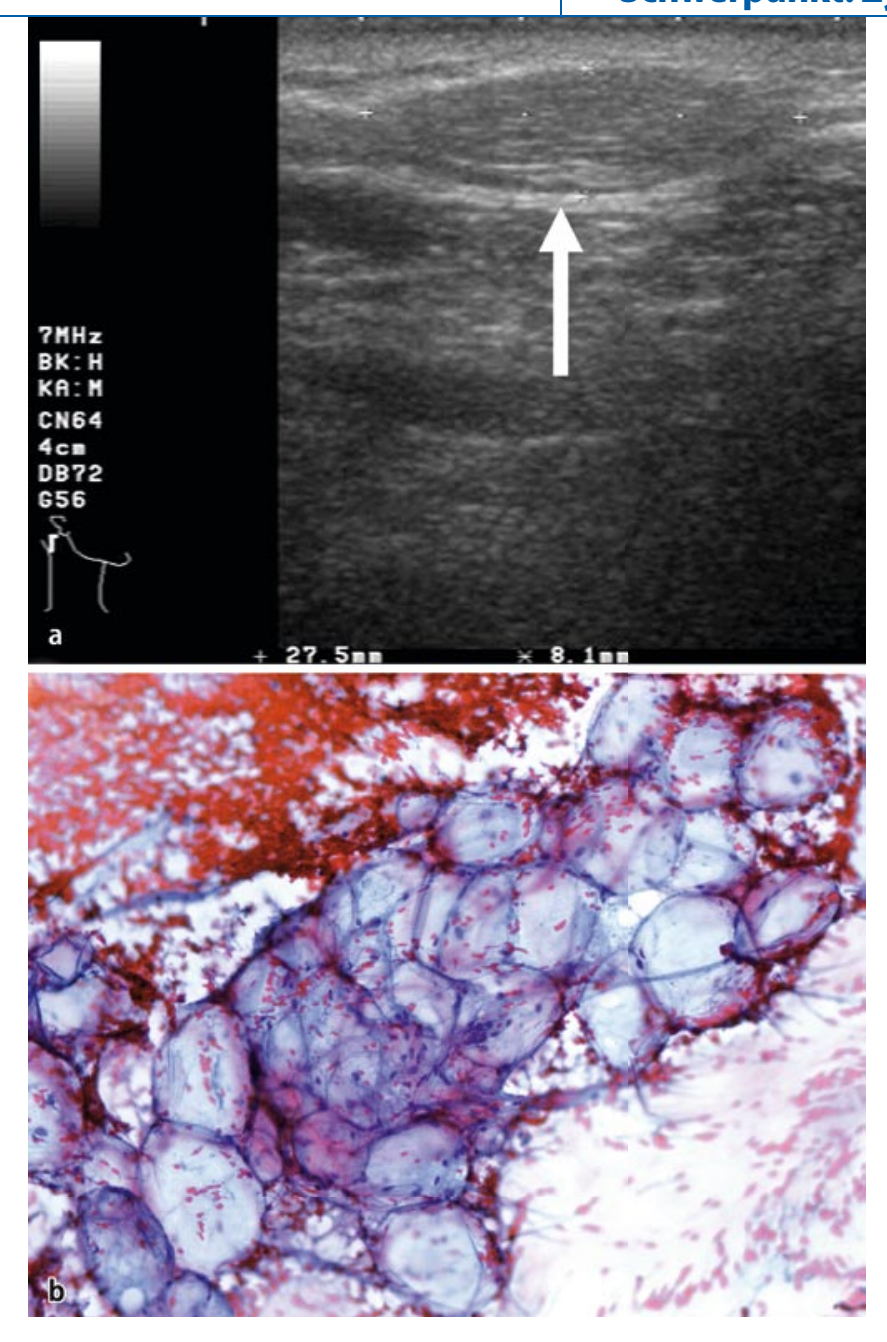

Abb. $5<$ Subkutanes Lipom bei einem 60jährigen Mann. a Die klinisch weiche, oberflächliche Schwellung stellt sich sonographisch als linsenförmige, subkutane Raumforderung dar (Pfeil). b FNP: ausschließlich Fragment von reifem Fettgewebe ohne Kernatypien (Papanicolaou-Färbung, Vergr. 1:400)
(Tumorosteoid), das Angiosarkom (Positivität für $\mathrm{CD}_{31}$ und/oder $\mathrm{CD}_{34}$ ) oder der MPNST (Zusammenhang mit Nerven) dar. Trotz des Einsatzes aller verfügbaren Hilfsmethoden verbleibt eine Subgruppe pleomorphzelliger Sarkome, die sich nicht weiter zuordnen lassen und gemäß der geltenden WHO-Klassifikation [10] als ,undifferentiated high grade pleomorphic sarcoma" bezeichnet werden.

Aus praktischen Gründen ist eine detaillierte Unterscheidung des Subtyps eines pleomorphzelligen, hoch malignen Sarkoms bei den zur Zeit zur Verfügung stehenden therapeutischen Optionen für das klinische Management des Patienten nicht immer von prioritärer Bedeutung und auch nicht immer aufgrund der histologischen Untersuchung einer Stanzbiopsie zu erreichen. Solche Tumoren werden primär operativ behandelt, so dass die definitive Subtypisierung am Resektat erfolgen kann.

\section{Runde Zellen}

Diese Gruppe umfasst hoch maligne Neoplasien vor allem des Kindesalters, die selten auch im Erwachsenenalter anzutreffen sind. Insbesondere bei Erwachsenen muss man an Manifestationen von kleinzelligen Karzinomen, Melanomen oder (meist bereits vordiagnostizierten) Lymphomen denken. In der Diagnostik der so genannten „klein-, blau- und rundzelligen“ Tumoren ist der Einsatz der Zusatzmethoden auch am histologischen Material in den meisten Fällen unverzichtbar. $\mathrm{Da}$ es sich bei diesen Läsionen meist um sehr zellreiches Material handelt, enthalten sowohl die Direktausstriche als auch der Zellblock reichlich Tumorgewebe für immunhistochemische und molekulargenetische Untersuchungen. Sollten sich im Untersuchungsmaterial neben rundzelligen Anteilen weitere Bestandteile nachweisen lassen, so kann bei myxoidem Gewebe mit verzweigten zarten Kapillaren die Diagnose eines rundzelligen Liposarkoms und bei chondroiden Anteilen ein mesenchymales Chorndosarkom vermutet werden. Bereits der konventionell histologisch erfolgte Nachweis von Glykogen sowie die Ergebnisse der Immunhistochemie für CD99, myogene Marker (Desmin, Myogenin, MyoD1), neuroendokrine Marker (Synaptophysin) und epitheliale Marker (Zytokeratin, EMA) können richtungsweisend für die Diagnose sein.

Aufgrund des zytologischen Befundes und des Immunprofils lassen sich gezielte molekulargenetische Untersuchungen einsetzen (• Tab. 2), die zur präzisen Diagnosestellung führen (• Abb. 4). Die Anwendung der Zusatzuntersuchungen bei den überwiegend aus runden Zellen bestehenden Weichteiltumoren führt [16] im höchsten Prozentsatz (bis zu 83\%) der Fälle im Vergleich mit anderen Grundmustern zu einer definitiven Subtypisierung.

\section{Epitheloide Zellen}

Große zytoplasmareiche, rund-ovaläre Zellen, die deutliche Zytoplasmagrenzen aufweisen und stellenweise kohäsive Zellformationen bilden, werden bei einigen seltenen Weichteilneoplasien beobachtet. Es handelt sich dabei häufig um epitheloide Varianten von unterschiedlichen Sarkomsubtypen, wie z. B. epitheloide Formen eines Angiosarkoms oder eines malignen peripheren Nervenscheidentumors, aber auch um eigenständige Tumorentitäten wie z. B. ein epitheloides Sarkom oder alveoläres Weichteilsarkom. Da neben diesen hochmalignen Sarkomen auch seltene benigne Tumoren (Granularzellzumor) oder selbstlimitierende pseudosarkomatöse Prozesse (proliferative Myositis) sich als überwiegend aus epitheloiden Zellen bestehende Veränderung präsentieren können, ist bei der Dignitätsbeurteilung Vorsicht geboten und die klinische Korrelation notwendig. Bei diesem morphologischen Erscheinungsbild ist es besonders wichtig, eine Karzinom-, Mesotheliom- bzw. Melanommetastase auszuschließen.

\section{Differenziertes Gewebe}

Die geweblichen Bestandteile der Läsionen aus dieser Gruppe sind zytomorpho- 
logisch einfach zu erkennen: Man findet Fragmente von reifem Fett- bzw. hyalinem Knorpelgewebe. Bei den lipomatösen Läsionen handelt es sich in der überwältigenden Mehrheit der Fälle um oberflächlich lokalisierte Knoten, die klinisch Lipomen entsprechen ( $\bullet$ Abb.5). Die Punktion erfolgt häufig auf Wunsch der $\mathrm{Pa}$ tienten, bei Größenzunahme bzw. zum Ausschluss von Metastasen bekannter Neoplasien. Die Abgrenzung zwischen unbedenklichen Lipomen und atypischen lipomatösen Tumoren/gut differenzierten Liposarkomen kann vor allem bei tief gelegenen Läsionen wegen der kleinherdigen Fettgewebsnekrosen mit reaktiven Atypien erschwert sein. Bei den im SchulterNacken-Bereich gelegenen lipomatösen Veränderungen mit Atypien muss man differenzialdiagnostisch die gutartigen Spindelzell- und pleomorphzelligen Lipome in Betracht ziehen, ehe ein Malignitätsverdacht geäußert wird.

\section{Bedeutung von \\ Zusatzuntersuchungen}

Der Zytopathologe ist im Grunde mit dem gleichen Gewebe und den gleichen Zellen konfrontiert wie der Histopathologe, der Stanzbiopsien bzw. Inzisionsbiopsien beurteilt. Bis auf einige technische Einzelheiten stehen ihm grundsätzlich die gleichen Zusatzuntersuchungen zur Verfügung $[11,14,16,28]$. Insbesondere der Einsatz der Zellblocktechnik lässt den scheinbaren Widerspruch zwischen einer FNP und einer Stanzbiopsie in vielen Fällen schwinden - die Bearbeitung, die diagnostischen Kriterien und die zur Verfügung stehende Methoden sind identisch $[27,30]$. In Bezug auf gewisse diagnostische Methoden, vor allem die FISH, ist jedoch der zytologische Ausstrich zu bevorzugen, da die schnittbedingten Artefakte eines histologischen Schnitts im dreidimensionalen Ausstrich entfallen.

\section{Grenzen in der Diagnostik der Weichteilneoplasien}

Der hohe Stellenwert der FNP in der Diagnostik von Rezidiven bzw. Metastasen klinisch bekannter Tumoren der Weichteile ist unbestritten. Bei der Diagnostik primärer Weichteilneoplasien ist jedoch die
Zytologie mit Einschränkungen konfrontiert, die auf die inhärenten Eigenschaften der untersuchten Läsionen zurückzuführen sind. Die stark kollagenhaltigen, zellarmen primären Weichteilläsionen, wie z. B. Fibromatosen oder niedrig maligne Sarkome, ergeben in einer FNP naturgemäß nur wenig Gewebe, während proliferative pseudosarkomatöse Prozesse vom Typ der nodulären Fasziitis recht zellreich sein können sowie zahlreiche Mitosen und ausgeprägte reaktive Atypien aufweisen $[4,31]$. Die spezifische Diagnose und präzise Dignitätseinschätzung kann in solchen Fällen schwierig sein, was jedoch nicht nur auf die zytologischen Proben, sondern auch für Stanzbiopsien zutrifft. Die morphologische Beurteilung solcher Proben erfordert eine sorgfaltige Korrelation mit dem klinischen Verlauf und der Bildgebung. Ähnlich wie die Stanzbiopsie oder sogar die Inzisionsbiopsie unterliegt die FNP dem Risiko eines gewissen „sampling error", was zu einer Fehleinschätzung der Dignität führen kann.

Die histologische Graduierung der Malignität eines Sarkoms gehört zu den wichtigsten prognostischen Faktoren. Die zytologisch durchgeführte Graduierung der Weichteilsarkome (unter Berücksichtigung der Kerneigenschaften, der Mitoserate und der Tumornekrose) korreliert gemäß einiger Studien gut mit der histologischen Graduierung nach dem FNCLCC-System mit einer Gradkonkordanz bis $74 \%[25,26]$. Problematisch sind jedoch methodenbedingte Artefakte, vor allem in Bezug auf die Einschätzung der Mitoserate oder der Ausdehnung der Nekrose. Der Stellenwert der zytologischen Graduierung wurde bisher nur in wenigen Studien belegt.

Zusammenfassend sind nicht nur die epithelialen Neoplasien, sondern auch die primären Weichteiltumoren zytologischer Diagnostik zugänglich. Ein multidisziplinärer Ansatz erlaubt die gezielte Anwendung moderner diagnostischer Methoden. Optimale Ergebnisse können erreicht werden, wenn der Zytopathologe entweder bei der Materialentnahme anwesend ist oder die FNP selbst durchführt. Die Diskussion mit dem Orthopäden und/oder Radiologen liefert ihm Anhaltspunkte zur Differenzialdiagnose, die gestützt auf den konventionellen zytologischen Befund und untermauert durch die molekularen $\mathrm{Zu}$ satzmethoden zu einer klinisch relevanten Aussage (Diagnose) führen kann.

\section{Fazit für die Praxis}

Die Feinnadelpunktion (FNP) stellt eine einfache, kostengünstige und komplikationsarme Methode in der Abklärung raumfordernder Prozesse der Weichteile dar. Zytomorphologisch lassen sich in den meisten Fällen entzündliche Veränderungen, Metastasen von Karzinomen und Melanomen bzw. lymphoproliferative Prozesse abgrenzen. Bei primär mesenchymalen Läsionen erlaubt eine Kombination von Zytologie mit modernen Zusatztechniken sowie den klinischen und radiologischen Befunden in vielen Fällen eine präzise Subtypisierung eines Tumors. Eine Unterscheidung zwischen benignen und malignen Läsionen ist bei adäquaten Proben fast immer möglich. Entscheidend für die Aussagekraft der FNP eines Weichteilprozesses ist eine hohe Qualität des Untersuchungsmaterials, Kenntnisse des Zytopathologen über die differenzialdiagnostischen Möglichkeiten und eine enge, multidisziplinäre Zusammenarbeit mit Orthopäden, Radiologen und Onkologen.

\section{Korrespondenzadresse}

\section{Dr. B. Bode-Lesniewska}

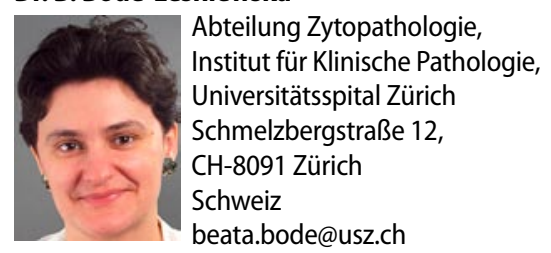

Interessenkonflikt. Der korrespondierende Autor gibt an, dass kein Interessenkonflikt besteht.

\section{Literatur}

1. Akerman M, Domanski HA (2003) The Cytology of Soft Tissue Tumours. Karger New York

2. Brown FM (2003) Soft tissue. In: Cibas ES, Ducatman BS (eds) Cytology. Diagnostic principles and clinical correlates. Saunders, Edinborough

3. Das K, Hameed M, Heller D et al. (2003) Liquid-based vs. conventional smears in fine needle aspiration of bone and soft tissue tumors. Acta Cytol 47: 197-201

4. Dodd LG, Martinez S (2001) Fine-needle aspiration cytology of pseudosarcomatous lesions of soft tissue. Diagn Cytopathol 24: 28-35

5. Domanski HA (2005) FNA diagnosis of dermatofibrosarcoma protuberans. Diagn Cytopathol 32: 299-302 
6. Domanski HA, Akerman M, Carlen B et al. (2005) Core-needle biopsy performed by the cytopathologist: a technique to complement fine-needle aspiration of soft tissue and bone lesions. Cancer 105: 229-239

7. Domanski HA, Akerman M, Engellau J et al. (2006) Fine-needle aspiration of neurilemoma (schwannoma). A clinicocytopathologic study of 116 patients. Diagn Cytopathol 34: 403-412

8. Domanski HA, Akerman M, Rissler P, Gustafson P (2006) Fine-needle aspiration of soft tissue leiomyosarcoma: An analysis of the most common cytologic findings and the value of ancillary techniques. Diagn Cytopathol 34: 597-604

9. Domanski HA, Gustafson P (2002) Cytologic features of primary, recurrent, and metastatic dermatofibrosarcoma protuberans. Cancer 96: 351-361

10. Fletcher CDM, Unni KK, Mertens F (eds) (2002) Tumours of Soft Tissue and Bone. IARC Press, Lyon

11. Frostad B, Tani E, Brosjo O et al. (2002) Fine needle aspiration cytology in the diagnosis and management of children and adolescents with Ewing sarcoma and peripheral primitive neuroectodermal tumor. Med Pediatr Oncol 38: 33-40

12. Geisinger KR, Abdul-Karim FW (2001) Fine needle aspiration biopsies of soft tissue tumors. In: Weiss SW, Goldblum JR (eds) Enzinger and Weiss, s Soft Tissue Tumors. Mosby, St. Louis London Philadelphia Sydney Toronto, pp 147-188

13. Gonzalez-Campora R (2000) Fine needle aspiration cytology of soft tissue tumors. Acta Cytol 44: 337-343

14. Kilpatrick SE, Bergman S, Pettenati MJ, Gulley ML (2006) The usefulness of cytogenetic analysis in fine needle aspirates for the histologic subtyping of sarcomas. Mod Pathol 19: 815-819

15. Kilpatrick SE, Ward WG, Bos GD (2000) The value of fine-needle aspiration biopsy in the differential diagnosis of adult myxoid sarcoma. Cancer 90: 167-177

16. Kilpatrick SE, Ward WG, Cappellari JO, Bos GD (1999) Fine-needle aspiration biopsy of soft tissue sarcomas. A cytomorphologic analysis with emphasis on histologic subtyping, grading, and therapeutic significance. Am J Clin Pathol 112: 179-188

17. Klijanienko J, Caillaud JM, Lagace R (2006) Cytohistologic correlations in schwannomas (neurilemmomas), including „, ancient," cellular, and epithelioid variants. Diagn Cytopathol 34: 517-522

18. Klijanienko J, Caillaud JM, Lagace R (2004) Fineneedle aspiration in liposarcoma: Cytohistologic correlative study including well-differentiated, myxoid, and pleomorphic variants. Diagn Cytopathol 30: 307-312

19. Klijanienko J, Caillaud JM, Lagace R, Vielh P (2002) Cytohistologic correlations in 56 synovial sarcomas in 36 patients: The Institut Curie experience. Diagn Cytopathol 27: 96-102

20. Klijanienko J, Caillaud JM, Lagace R, Vielh P (2003) Cytohistologic correlations in angiosarcoma including classic and epithelioid variants: Institut Curie's experience. Diagn Cytopathol 29: 140-145

21. Klijanienko J, Caillaud JM, Lagace R, Vielh P (2002) Cytohistologic correlations of 24 malignant peripheral nerve sheath tumor (MPNST) in 17 patients: The Institut Curie experience. Diagn Cytopathol 27: 103-108

22. Klijanienko J, Caillaud JM, Lagace R, Vielh P (2003) Fine-needle aspiration of leiomyosarcoma: A correlative cytohistopathological study of 96 tumors in 68 patients. Diagn Cytopathol 28: 119-125

23. Layfield LJ (ed) (2002) Cytopathology of bone an soft tissue tumors. Oxford University Press, Oxford

24. Maitra A, Ashfaq R, Saboorian MH et al. (2000) The role of fine-needle aspiration biopsy in the primary diagnosis of mesenchymal lesions: A community hospital-based experience. Cancer 90: 178-185
25. Mathur S, Kapila K, Verma K (2003) Accuracy of cytological grading of spindle-cell sarcomas. Diagn Cytopathol 29: 79-83

26. Palmer HE, Mukunyadzi P, Culbreth W, Thomas JR (2001) Subgrouping and grading of soft-tissue sarcomas by fine-needle aspiration cytology: A histopathologic correlation study. Diagn Cytopathol 24: 307-316

27. Pfeiffer DF, Bode-Lesniewska B (2006) Fine needle aspiration biopsy diagnosis of angiosarcoma after breast-conserving therapy for carcinoma supported by use of a cell block and immunohistochemistry. Acta Cytol 50: 553-556

28. Sapi Z, Antal I, Papai Z et al. (2002) Diagnosis of soft tissue tumors by fine-needle aspiration with combined cytopathology and ancillary techniques. Diagn Cytopathol 26: 232-242

29. Wakely PE Jr, Bos GD, Mayerson J (2005) The cytopathology of soft tissue mxyomas: Ganglia, juxta-articular myxoid lesions, and intramuscular myxoma. Am J Clin Pathol 123: 858-865

30. Wakely PE, Kneisl JS (2000) Soft tissue aspiration cytopathology. Cancer 90: 292-298

31. Wong NL (2002) Fine needle aspiration cytology of pseudosarcomatous reactive proliferative lesions of soft tissue. Acta Cytol 46: 1049-1055

\section{Preise der Konrad-Morgenroth- Fördergesellschaft e.V.}

Die Konrad-Morgenroth-Fördergesellschaft e.V.(KMFG) vergibt für den Zeitraum 2007/2008 die von ihr gestifteten beiden Preise in Höhe von jeweils 3.500,- Euro.

Diese werden verliehen für bisher noch nicht publizierte Arbeiten auf dem Gebiet der Grundlagenforschung, Vorbeugung und Behandlung bösartiger Geschwülste im Mundhöhlen- und Kieferbereich sowie auf jenem der zahnärztlichen Behandlung spastisch $\mathrm{Ge}$ lähmter und/oder geistig Behinderter, auch im Hinblick auf anästhesiologische Belange. Darüber hinaus können auch abgeschlossene Promotionsarbeiten mit gleicher Thematik vorgelegt werden.

Teilnahmeberechtigt ist jeder in Deutschland tätige Zahnarzt, Arzt oder Naturwissenschaftler. Die Arbeiten sind in dreifacher Ausfertigung bis zum 31. Dezember 2008 einzureichen bei der

Geschäftsstelle der KMFG,

Auf der Horst 29,

48147 Münster.

Sie sind mit einem Kennwort zu versehen und dürfen den Verfasser nicht erkennen lassen. Die Anschrift des Autors ist in einem gesonderten, mit dem Kennwort bezeichneten, verschlossenen Umschlag beizulegen.

Die Arbeiten werden vom Wissenschaftsbeirat der KMFG beurteilt. Der Vorstand entscheidet mehrheitlich nach dessen Vorschlägen. Die Entscheidung des Vorstandes ist bindend, der Rechtsweg ausgeschlossen.

Quelle: Konrad-Morgenroth-Fördergesellschaft e.V. 